\title{
Hybrid Motion Reproduction Using Master/Slave Type Motion-Copying System
}

\author{
Satoshi Nishimura* Member, Seiichiro Katsura* Senior Member
}

(Manuscript received May 1, 2018, revised April 7, 2019)

\begin{abstract}
This paper proposes a hybrid motion reproduction structure using a master-slave structure manipulator based on a motion-copying system. Data-driven control will be an important technique in the near future. Learning from Demonstration (LfD) using a robot manipulator is already used in manufacturing. Unlike other motion observation methods based on visual or trajectory responses, a motion-copying system can obtain not only trajectory response but also force response during contact motion, which is necessary to realize contact tasks. A motion-copying system has two phases, the motion saving phase and motion reproduction phase. In the motion reproduction phase, the motion stored in the motion saving phase obtained from the bilateral control can be used to reproduce specific motion. Existing control methods for a motion-copying system have only one system in the reproduction phase, a slave system. It is necessary to consider the case where there are two systems in the phase to widen the application of a motion-copying system. The additional system can provide flexibility to the reproduced motion. In this case, data, human, and a robot are involved in reproducing the motion. In this paper, the control structure is proposed when there are two systems in the motion reproduction phase. Because there are two systems, it is necessary to design the controllers for each system. The proposed method uses a coordinate transformation technique to design two motions in each system separately. The paper also introduces a novel index called the reproduction ratio. It determines the motion to be reproduced in the motion reproduction phase. The ratio is used as an element of a coordinate transformation matrix. The experimental results show the validity of the proposed method.
\end{abstract}

Keywords: acceleration control, position control, force control, modal transformation, motion-copying system

\section{Introductions}

In recent years, motion transfer technique to robot manipulators is attracting attention. By realizing the technique, it can be expanded to many applications. To name a few, it can be used in manufacturing factories, education sites, etc. If robots can mimic the motion of human workers in factories, the number of workers can be reduced. Or, if a robot remembers the movement of a skilled person, the robot becomes a teacher that can teach the motion to trainees.

There can be two ways to transfer motion to robots; the first ways is to transmit and use it in realtime, while the other way is to use the motion afterward. Bilateral control is a useful technique to transmit haptic sensation between two places, consequently transferring motion to remote robots. Research of bilateral control has been done since the 1940s. Goertz et al. proposed the first bilateral mechanism ${ }^{(1)}$. Since the birth of bilateral control, numbers of control structures were introduced to transmit haptic sensation clearly ${ }^{(2)}$. To name a few, there are force reflecting structure ${ }^{(3)}$, impedance reflecting structure ${ }^{(4)}$, two-channel structure ${ }^{(5)}$, three-channel structure ${ }^{(6)}$, etc. Transparency is one of the famous indices to examine haptic sensation transmission performance ${ }^{(7)}$. It views the system as a two-port circuit, and the relationship between control variables represented by transfer functions are used

\footnotetext{
* Department of System Design Engineering, Keio University

3-14-1, Hiyoshi, Kohoku-ku, Yokohama 223-8522, Japan
}

to show how precisely the sensation can be sent. Those are more specific indices to express how light the system is and how well the impedance of a contact object is reproduced in a remote system by dividing the transfer function in contact motion into two. Among many structures, acceleration-based four channel bilateral control has the best transparency or operationality and reproducibility ${ }^{(8)}$.

The other way of using the motion is to utilize after making robots learn the motion. There are several ways to obtain a demonstration motion. Motion storing using visual information is one way to observe human motion ${ }^{(9)}$; however, it is very difficult to analyze contact motions because it is very difficult to extract contact force during the motion. There are other ways to obtain human motion information, such as using IMU sensors; however, the problem still exists. A motion-copying system is a powerful tool to store motion with haptic sensation ${ }^{(10)}$. It solves the problem by using a bilateral control system to obtain not only trajectory information but also information of contact force. A motioncopying system consists of two phases. The first phase is a motion storing phase, and the other is a motion reproduction phase. The motion is stored by obtaining the response of the master system in bilateral control. Then, the responses are loaded to a system to reproduce the stored movement. Its application area is very wide. Nowadays, there are many robot manipulators such as Baxter ${ }^{(11)}$ that can be used for motion training once the motion is obtained. It can be used to service robots for them to realize more human-like motion ${ }^{(12)}$. 
Control of data-driven systems ${ }^{(13)}$ has become to attract attention, meaning that there will be an increasing opportunity for the motion-copying system to be an essential technique for these systems. The motion obtained can also be used to analyze human motion ${ }^{(14)}$.

One of the challenging problems in a motion-copying system is to improve the flexibility of the stored motion. Examples of flexibility mean adapting to a loading environment when it is different from the one in the saving phase, or expanding the situation to use the stored motion itself. Discussion about the adaptiveness has been done in many papers. The adaptability to loading environments can be improved by changing control stiffness during the motion reproduction phase $^{(15)}$. Mode-based reproduction was also considered ${ }^{(16)}$ to make the problem similar to that in bilateral control. Position control and force control based motion-copying systems were proposed ${ }^{(17)}$ so that the control stiffness can be designed from zero to infinite. The report ${ }^{(18)}$ defines control stiffness when reproducing motion, indicating that the value can be designed from zero to infinite by changing the control structure in the motion reproduction phase.

Even though there are researches to improve the reproduction performance in a motion-copying system, there are very few reports that discuss to use a master/slave type manipulator in the motion reproduction phase. By using the master/slave type manipulator, it can obtain both forces independently based on the fact from bilateral control. The master/slave structure gives the flexibility to a standard motioncopying system. For example, an operator in the master system can provide the adaptability to the saved motion because a human has higher adaptability than robots. It can also be implemented to a training system when storing the skilled motion and learning the movement using the master system. Data/human hybrid motion reproduction is required to be considered. However, since the number of actuator increases, the connectivity of each system becomes complicated. In this case, two problems are required to be solved: what kind of constraint should be given to the master system, and what motion to reproduce in the slave system.

In this paper, the hierarchal coordinate transformation technique is used to design two problems separately. Transforming saved and reproducing master responses to another coordinate realizes to consider the average motion and the motion difference between the two. The difference between the saved motion and the motion being performed at the master system in the motion reproduction phase is considered to give a constraint to the master system. The average motion is used to reproduce the motion in the slave system. To define the average motion, the index called the reproduction ratio is newly defined. The reproduction ratio is defined as the influence of external force in motion saving and reproduction phase on the trajectory response to the slave system. The design of the reproduction ratio realizes hybrid motion reproduction.

The paper is organized as follows. In section 2, bilateral control and a standard motion-copying system are shown. In section 3, the proposed structure is derived. Section 4 explains the experimental results to show the validity of the proposed method, and the paper is concluded in the last.

\section{Motion-Copying System}

As it is mentioned in the Introduction, a motion-copying system consists of two phases: the motion saving phase and motion reproduction phase. This section explains both phases in turn. The paper uses a disturbance observer (DOB) ${ }^{(19)}$ for acceleration dimension control and a reaction force observer $(\mathrm{RFOB})^{(20)}$ for sensor-less external force estimation. The paper also assumes that the cut-off frequencies of the DOB and RFOB are high enough.

2.1 Motion Saving Phase Human motion is going to be obtained by using bilateral control. Control goals of bilateral control are defined as

$$
\begin{aligned}
& f_{\mathrm{m}}^{\mathrm{DB}}+f_{\mathrm{s}}^{\mathrm{DB}}=0, \\
& x_{\mathrm{m}}^{\mathrm{DB}}-x_{\mathrm{s}}^{\mathrm{DB}}=0,
\end{aligned}
$$

where $f$ and $x$ stand for force and position responses, respectively. The subscripts $\mathrm{m}$ and $\mathrm{s}$ stand for the variables for the master and slave systems. The superscript DB indicates the variable in the motion saving phase. To realize each control goal independently, the values are mapped from the workspace to a modal space by using a coordinate transformation matrix, which is called a quarry matrix. The quarry matrix is

$$
\boldsymbol{Q}=\frac{1}{2}\left[\begin{array}{rr}
1 & 1 \\
1 & -1
\end{array}\right]
$$

The relationship between variables in the workspace and those in the modal space is expressed as

$$
\begin{aligned}
& \boldsymbol{f}_{\mathrm{v}}^{\mathrm{DB}}=\boldsymbol{Q} \boldsymbol{f}^{\mathrm{DB}}, \\
& \boldsymbol{x}_{\mathrm{v}}^{\mathrm{DB}}=\boldsymbol{Q} \boldsymbol{x}^{\mathrm{DB}} \text {, } \\
& \boldsymbol{f}_{\mathrm{v}}^{\mathrm{DB}}=\left[f_{\mathrm{c}}^{\mathrm{DB}} f_{\mathrm{d}}^{\mathrm{DB}}\right]^{T}, \boldsymbol{f}^{\mathrm{DB}}=\left[f_{\mathrm{m}}^{\mathrm{DB}} f_{\mathrm{s}}^{\mathrm{DB}}\right]^{T} \text {, } \\
& \boldsymbol{x}_{\mathrm{v}}^{\mathrm{DB}}=\left[\begin{array}{ll}
x_{\mathrm{c}}^{\mathrm{DB}} & x_{\mathrm{d}}^{\mathrm{DB}}
\end{array}\right]^{T}, \boldsymbol{x}^{\mathrm{DB}}=\left[\begin{array}{ll}
x_{\mathrm{m}}^{\mathrm{DB}} & x_{\mathrm{s}}^{\mathrm{DB}}
\end{array}\right]^{T},
\end{aligned}
$$

where the subscripts $\mathrm{v}$ indicates the vector of the modal space. The subscripts $c$, and d, which are called as the variables of the common mode and differential mode, stand for the variable in the modal space, respectively. Acceleration references for both modes are calculated as

$$
\begin{aligned}
& \ddot{x}_{\mathrm{c}}^{\mathrm{DB} \text { ref }}=C_{\mathrm{f}}\left(f_{\mathrm{c}}^{\mathrm{cmd}}-f_{\mathrm{c}}^{\mathrm{DB}}\right), \\
& \ddot{x}_{\mathrm{d}}^{\mathrm{DB} \text { ref }}=C_{\mathrm{p}}\left(x_{\mathrm{d}}^{\mathrm{cmd}}-x_{\mathrm{d}}^{\mathrm{DB}}\right),
\end{aligned}
$$

where $C_{\mathrm{f}}$ and $C_{\mathrm{p}}$ stand for the force and position controllers, respectively. $C_{\mathrm{f}}$ is the $\mathrm{P}$ controller, while $C_{\mathrm{p}}$ is the PD controller. The superscript cmd and ref stand for the command value and reference value. The command values are $f_{\mathrm{c}}^{\mathrm{cmd}}=0, x_{\mathrm{d}}^{\mathrm{cmd}}=0$. Acceleration references for each system are calculated by retransforming (4) and (5), which are the values in the modal space, into the workspace by using the inverse quarry matrix. Acceleration reference $\ddot{\boldsymbol{x}}^{\mathrm{DB} \text { ref }}$ is finally derived as

$$
\ddot{\boldsymbol{x}}^{\mathrm{DB} \text { ref }}=-C_{\mathrm{f}} \boldsymbol{Q}^{-1} \boldsymbol{S}_{\mathrm{f}} \boldsymbol{Q} \boldsymbol{f}^{\mathrm{DB}}-C_{\mathrm{p}} \boldsymbol{Q}^{-1} \boldsymbol{S}_{\mathrm{p}} \boldsymbol{Q} \boldsymbol{x}^{\mathrm{DB}}, \cdots \cdots
$$

where $\ddot{\boldsymbol{x}}^{\mathrm{DB} \text { ref }}, \mathcal{S}_{\mathrm{f}}$, and $\mathcal{S}_{\mathrm{p}}$ are

$$
\begin{aligned}
& \ddot{\boldsymbol{x}}^{\mathrm{DB} \text { ref }}=\left[\ddot{x}_{\mathrm{m}}^{\mathrm{DB} \text { ref }} \ddot{x}_{\mathrm{s}}^{\mathrm{DB} r e f}\right], \\
& \mathcal{S}_{\mathrm{f}}=\operatorname{diag}\left[\begin{array}{ll}
1 & 0
\end{array}\right], \cdots \cdots \cdots \\
& \mathcal{S}_{\mathrm{p}}=\operatorname{diag}\left[\begin{array}{ll}
0 & 1
\end{array}\right] . \cdots \cdots \cdots
\end{aligned}
$$

Figure 1 shows the block diagram of the motion saving phase. 


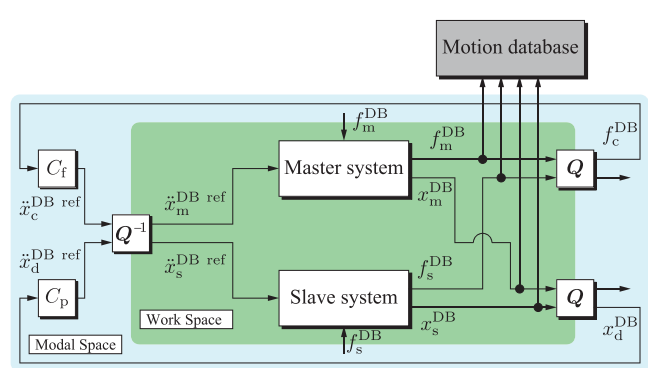

Fig. 1. Block diagram of motion saving phase
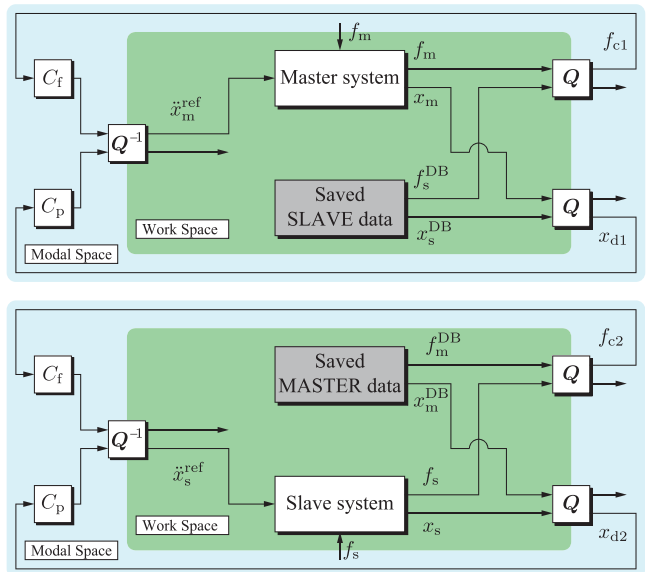

Fig. 2. Block diagram of motion reproduction phase using master and slave systems

2.2 Motion Reproduction Phase-Slave System-

Once the motions are stored into a database, the data can be used to reproduce the motion. The control structure of the reproduction phase is similar to that of the motion saving phase. In this phase, the vectors are defined as

$$
\boldsymbol{f}=\left[\begin{array}{ll}
f_{\mathrm{m}}^{\mathrm{DB}} & f_{\mathrm{s}}
\end{array}\right]^{T}, \boldsymbol{x}=\left[\begin{array}{ll}
x_{\mathrm{m}}^{\mathrm{DB}} & x_{\mathrm{s}}
\end{array}\right]^{T} .
$$

The acceleration reference for the slave system is calculated in the same manner as

$$
\ddot{\boldsymbol{x}}^{\mathrm{ref}}=-C_{\mathrm{f}} \boldsymbol{Q}^{-1} \boldsymbol{S}_{\mathrm{f}} \boldsymbol{Q} \boldsymbol{f}-C_{\mathrm{p}} \boldsymbol{Q}^{-1} \boldsymbol{S}_{\mathrm{p}} \boldsymbol{Q} \boldsymbol{x}
$$

2.3 Motion Reproduction Phase-Master and Slave Systems- There can be a lot of structures considered when there are master and slave systems in the motion reproduction phase. One of the structures is to use both responses of stored master and slave systems in this phase. The structure used in this paper as a conventional method is shown in Fig. 2. The concept of this system is simple; stored motion of the master system is loaded at the slave system, while stored motion of the slave system used in the master system.

The workspace variables used in the modal space are defined as

$$
\begin{aligned}
& \boldsymbol{f}_{1}=\left[f_{\mathrm{m}}^{\mathrm{DB}} f_{\mathrm{s}}\right]^{T}, \boldsymbol{x}_{1}=\left[x_{\mathrm{m}}^{\mathrm{DB}} x_{\mathrm{s}}\right]^{T}, \\
& \boldsymbol{f}_{2}=\left[f_{\mathrm{m}} f_{\mathrm{s}}^{\mathrm{DB}}\right]^{T}, \boldsymbol{x}_{2}=\left[x_{\mathrm{m}} x_{\mathrm{s}}^{\mathrm{DB}}\right]^{T},
\end{aligned}
$$

and the modal space variables are given as

$$
\begin{aligned}
\boldsymbol{f}_{\mathrm{v} i} & =\boldsymbol{Q} \boldsymbol{f}_{i}, \\
\boldsymbol{x}_{\mathrm{v} i} & =\boldsymbol{Q} \boldsymbol{x}_{i}, \\
\boldsymbol{f}_{\mathrm{v} i} & =\left[f_{\mathrm{c} i} f_{\mathrm{d} i}\right]^{T}, \boldsymbol{x}_{\mathrm{v} i}=\left[\begin{array}{ll}
x_{\mathrm{c} i} & x_{\mathrm{d} i}
\end{array}\right]^{T} \quad(i=1,2) .
\end{aligned}
$$

The acceleration references for this system is calculated as

$$
\ddot{\boldsymbol{x}}_{i}^{\mathrm{ref}}=-C_{\mathrm{f}} \boldsymbol{Q}^{-1} \boldsymbol{S}_{\mathrm{f}} \boldsymbol{Q} \boldsymbol{f}_{i}-C_{\mathrm{p}} \boldsymbol{Q}^{-1} \boldsymbol{S}_{\mathrm{p}} \boldsymbol{Q} \boldsymbol{x}_{i}(i=1,2) .
$$

Acceleration reference matrices are $\ddot{x}_{1}^{\text {ref }}=\left[\ddot{x}_{\mathrm{m}}^{\text {ref }} \circ\right], \ddot{x}_{2}^{\text {ref }}=$ $\left[\circ \ddot{\boldsymbol{x}}_{\mathrm{s}}^{\mathrm{ref}}\right]$ where $\circ$ is the variable not used in the control. The important point is that there is no connection between the master and slave systems in this structure.

\section{Proposed Control Structure}

In this section, the proposed method is introduced.

3.1 Proposed Quarry Matrix As explained in the Introduction, the paper uses hierarchal coordinate transformation. Firstly, the block diagram is shown in Fig. 3. The matrix $\boldsymbol{T}$ is newly introduced to realize hybrid motion reproduction. To achieve hybrid motion reproduction, two problems have to be solved. They are, what kind of constraint should be given to the master system, and what motion to reproduce in the slave system. Introducing the coordinate transformation technique based on the quarry matrix gives the answer to both of them.

As for the first problem, it can see the difference between the saved master motion and the master motion in the reproducing phase by subtracting position and force responses of both motions. It can be used to directly give constraint to the motion of the master system in the reproduction phase.

As for the second problem, what motion is reproduced in the slave system can be determined by what response to transforming to the modal space with the response of the slave system. The common mode can be viewed as the summation of both motions. The reproduction ratio is newly introduced to design how to sum the motions. Reproduction ratio is defined as the influence of external force in motion saving and reproduction phase on the trajectory response to the slave system. It is used as a scaling gain. Since the paper treats two master systems, two ratios are used that are defined as $\alpha$ and $\beta$. $\alpha$ and $\beta$ become the ratio of mixing the master system's motion in the saving and reproduction phase. The derived coordinate transformation matrix $\boldsymbol{T}$ is given as follows:

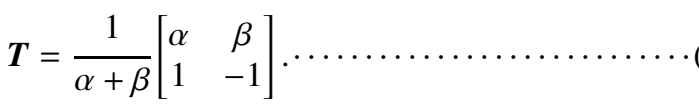

$\alpha$ defines the reproduction ratio regarding the saved motion, and $\beta$ defines the ratio of the motion of the master system in the reproduction phase. The ratio is defined to take the following values:

$$
\begin{aligned}
& 0 \leq \alpha \leq 1, \\
& 0 \leq \beta \leq 1 .
\end{aligned}
$$

The relationship of setting $\alpha$ and $\beta$, and how the motion is reproduced is explained in Table 1. Some examples are given in the following:

$(\alpha, \beta)=(1,0):$ only the saved motion is reproduced. ................ (18)

$\alpha \neq 0, \beta \neq 0$ : both of the motions are reproduced.

$(\alpha, \beta)=(0,1):$ only the master motion of the 


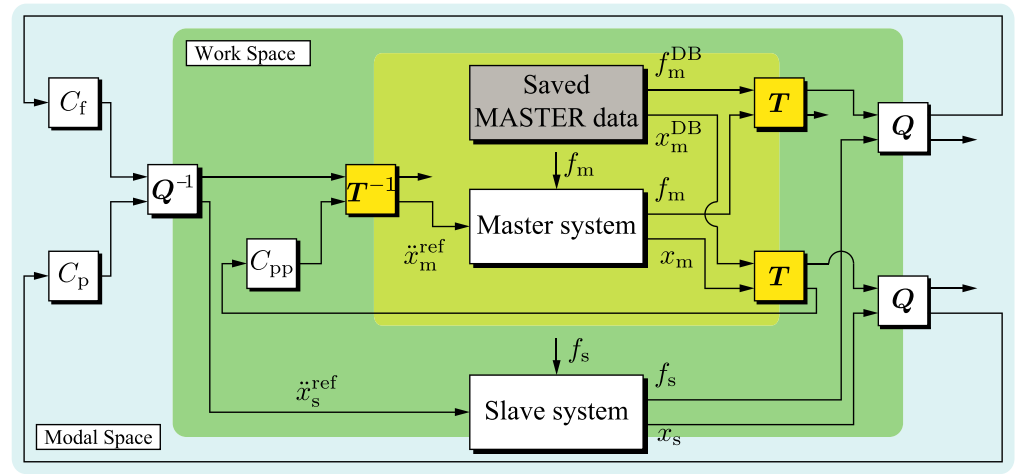

Fig. 3. Block diagram of the proposed method

Table 1. Reproduction ratio

\begin{tabular}{|c|c|}
\hline Condition & Reproduction Ratio $\alpha$ and $\beta$ \\
\hline \hline fully reproduced & 1 \\
reproduced & $0<\alpha<1,0<\beta<1$ \\
not reproduced & 0 \\
\hline \hline
\end{tabular}

Table 2. Parameters for analysis

\begin{tabular}{|c|l|c|}
\hline Parameter & Description & Value \\
\hline \hline$K_{\mathrm{p}}$ & P gain in position control & 10000.0 \\
$K_{\mathrm{d}}$ & D gain in position control & 200.0 \\
$C_{\mathrm{f}}$ & P gain in force control & 1.0 \\
\hline \hline
\end{tabular}

reproduction phase is reproduced.

The acceleration reference of each system is obtained as

$$
\begin{aligned}
\ddot{x}_{\mathrm{m}}^{\mathrm{ref}}= & \frac{\alpha}{\alpha+\beta}\left(x_{\mathrm{m}}^{\mathrm{DB}}-x_{\mathrm{m}}\right) C_{\mathrm{pp}}-\frac{1}{2}\left(\frac{\alpha f_{\mathrm{m}}^{\mathrm{DB}}+\beta f_{\mathrm{m}}}{\alpha+\beta}+f_{\mathrm{s}}\right) C_{\mathrm{f}} \\
& -\frac{1}{2}\left(\frac{\alpha x_{\mathrm{m}}^{\mathrm{DB}}+\beta x_{\mathrm{m}}}{\alpha+\beta}-x_{\mathrm{s}}\right) C_{\mathrm{p}} \ldots \ldots \ldots \ldots \ldots \ldots \ldots \ldots \ldots \ldots \\
\ddot{x}_{\mathrm{s}}^{\mathrm{ref}}= & -\frac{1}{2}\left(\frac{\alpha f_{\mathrm{m}}^{\mathrm{DB}}+\beta f_{\mathrm{m}}}{\alpha+\beta}+f_{\mathrm{s}}\right) C_{\mathrm{f}}-\frac{1}{2}\left(x_{\mathrm{s}}-\frac{\alpha x_{\mathrm{m}}^{\mathrm{DB}}+\beta x_{\mathrm{m}}}{\alpha+\beta}\right) C_{\mathrm{p}},
\end{aligned}
$$

where $C_{\mathrm{pp}}$ is the PD controller. $C_{\mathrm{pp}}$ is used to constrain the trajectory of the master system in the motion reproduction phase.

3.2 Connectivity Analysis The relationship between the variables in the loading phase is analyzed in the following. The paper assumes three cases to analyze the connectivity:

$$
\begin{array}{ll}
\text { Case 1: } & (\alpha, \beta)=(1,0), C_{\mathrm{pp}} \neq 0, \\
\text { Case 2: } & (\alpha, \beta)=(1,1), C_{\mathrm{pp}}=0, \\
\text { Case 3: } & (\alpha, \beta)=(0,1), C_{\mathrm{pp}}=0 .
\end{array}
$$

The connectivity matrix is defined as

$$
\begin{aligned}
& {\left[\begin{array}{c}
x_{\mathrm{m}} \\
x_{\mathrm{s}}
\end{array}\right]=\boldsymbol{H}\left[\begin{array}{llll}
-f_{\mathrm{m}}^{\mathrm{DB}} & -f_{\mathrm{m}} & -f_{\mathrm{s}} & x_{\mathrm{m}}^{\mathrm{DB}}
\end{array}\right]^{T} \text {, }} \\
& \boldsymbol{H}=\frac{1}{\alpha A\left(2 s^{2}+C_{\mathrm{p}}\right)+2 \beta B s^{2}}
\end{aligned}
$$

$$
\begin{aligned}
& {\left[\begin{array}{cc}
\alpha C_{\mathrm{f}} B & \frac{\alpha C_{\mathrm{f}}(\alpha A+\beta B)}{\alpha+\beta} \\
\beta C_{\mathrm{f}} B & \frac{\beta C_{\mathrm{f}}(\alpha A+\beta B)}{\alpha+\beta} \\
(\alpha+\beta) C_{\mathrm{f}} B & C_{\mathrm{f}}(\alpha A+\beta B) \\
\alpha\left(C_{\mathrm{p}} A+\left(C_{\mathrm{pp}}-C_{\mathrm{p}}\right) 2 s^{2}\right) & \alpha C_{\mathrm{p}} A
\end{array}\right]^{T}, \cdots(2)} \\
& A=s^{2}+C_{\mathrm{pp}}, B=s^{2}+C_{\mathrm{p}} .
\end{aligned}
$$

The reason for the structure of $\boldsymbol{H}$ is because to analyze how the trajectories of the systems in the motion reproduction phase were affected by other variables.

The connectivity matrices of each case are calculated as

$$
\begin{aligned}
& \boldsymbol{H}_{1}=\frac{1}{A\left(2 s^{2}+C_{\mathrm{p}}\right)}\left[\begin{array}{cc}
C_{\mathrm{f}} B & C_{\mathrm{f}} A \\
0 & 0 \\
C_{\mathrm{f}} B & C_{\mathrm{f}} A \\
C_{\mathrm{p}} A+\left(C_{\mathrm{pp}}-C_{\mathrm{p}}\right) 2 s^{2} & C_{\mathrm{p}} A
\end{array}\right]^{T} \\
& \boldsymbol{H}_{2}=\frac{1}{s^{2}\left\{\left(2 s^{2}+C_{\mathrm{p}}\right)+2 B\right\}}\left[\begin{array}{cc}
C_{\mathrm{f}} B & \frac{C_{\mathrm{f}}\left(s^{2}+B\right)}{2} \\
C_{\mathrm{f}} B & \frac{C_{\mathrm{f}}\left(s^{2}+B\right)}{2} \\
2 C_{\mathrm{f}} B & C_{\mathrm{f}}\left(s^{2}+B\right) \\
C_{\mathrm{p}} s^{2} & C_{\mathrm{p}} s^{2}
\end{array}\right]^{T}, \\
& \text {................. (26) } \\
& \boldsymbol{H}_{3}=\frac{C_{\mathrm{f}}}{2 s^{2}}\left[\begin{array}{llll}
0 & 1 & 1 & 0 \\
0 & 1 & 1 & 0
\end{array}\right],
\end{aligned}
$$

where the subscript shows the number of the case.

3.3 Structural Analysis and Controller Design of $\boldsymbol{C}_{\mathrm{pp}}$ in Case 1 The second row of $\boldsymbol{H}_{1}$ becomes 0, which means the force applied to the master system in the motion reproduction phase will not affect the trajectory of both systems. This is because the ratio $\beta$ is 0 in this case.

How to set the gain of $C_{\mathrm{pp}}$ will be explained in the following. The bode diagram of $H_{14}$ and $H_{24}$ when changing $K_{\mathrm{pp}}$ from 0.0 to $10000.0\left(C_{\mathrm{pp}}=K_{\mathrm{pp}}+2 \sqrt{K_{\mathrm{pp}}} s\right)$ is shown in Fig. 4. When $K_{\mathrm{pp}}$ is 0 , although the amplitude at low frequency is $0 \mathrm{~dB}$, the phase becomes -180 degree. It indicates that the trajectory response of the saved master system has effect to the opposite direction between the master and slave systems in the motion reproduction phase. Moreover, when $K_{\text {pp }}$ is smaller than $K_{\mathrm{p}}$, the phase exceeds -180 degree at a specific frequency. However, when the gains are the same, 

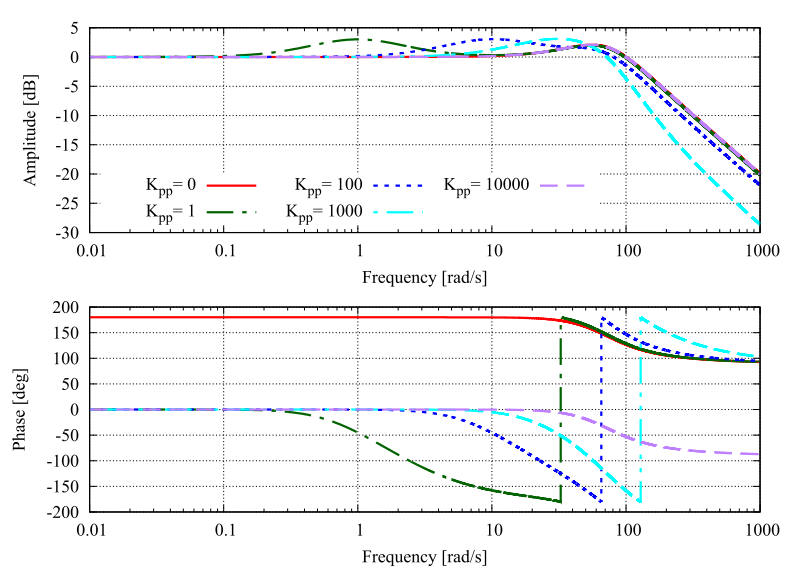

Fig. 4. Bode plot of $H_{14}$ in Case 1

the phase does not cross -180 degree. It indicates that $K_{\mathrm{pp}}$ should be set as same as $K_{\mathrm{p}}$. When setting $C_{\mathrm{pp}}=C_{\mathrm{p}}$, (25) becomes

$$
\boldsymbol{H}_{1}=\frac{1}{2 s^{2}+C_{\mathrm{p}}}\left[\begin{array}{llll}
C_{\mathrm{f}} & 0 & C_{\mathrm{f}} & C_{\mathrm{p}} \\
C_{\mathrm{f}} & 0 & C_{\mathrm{f}} & C_{\mathrm{p}}
\end{array}\right]
$$

The elements become the same as the motion-copying system with only a slave system.

3.4 Structural Analysis of Case 2 In Case 2, all the elements have frequency characteristics. $H_{21}$ and $H_{22}\left(H_{11}\right.$ and $H_{12}$ ) have the same element, meaning that the amount of the effect to the slave reproduced motion (constraint to the master system) between the saved force and the force applied at the master system in the motion reproduction phase is the same. It can be said that the proposed structure covers from bilateral control to motion-copying system by designing $\alpha$ and $\beta$.

Comprehensive analysis is conducted in the following. By modifying (26) with remaining $\alpha$ and $\beta$ as a variable and combining two equations into one, the following equation can be obtained:

$$
\begin{aligned}
x_{\mathrm{s}}= & \frac{2 A B(\alpha A+\beta B) C_{\mathrm{f}}}{\left\{\alpha A\left(2 s^{2}+C_{\mathrm{p}}\right)+2 \beta B s^{2}\right\}\left(2 s^{2}+C_{\mathrm{p}}\right)}\left(f_{\mathrm{cpT}}+f_{\mathrm{s}}\right) \\
& +\frac{C_{\mathrm{p}}}{2 s^{2}+C_{\mathrm{p}}} x_{\mathrm{cpT}}, \cdots \ldots \ldots \ldots \ldots \ldots \ldots \ldots \ldots \ldots \ldots \ldots \ldots
\end{aligned}
$$

where

$$
x_{\mathrm{cpT}}=\frac{\alpha x_{\mathrm{m}}^{\mathrm{DB}}+\beta x_{\mathrm{m}}}{\alpha+\beta}, f_{\mathrm{cpT}}=\frac{\alpha f_{\mathrm{m}}^{\mathrm{DB}}+\beta f_{\mathrm{m}}}{\alpha+\beta} .
$$

$x_{\mathrm{m}}$ is also included in the right-hand side of (29) to see the response of all the variables. It indicates that the trajectory of the slave system in the motion reproduction phase will track that of the mixed master systems, and the law of action and reaction between the slave force and the combined master forces can be achieved.

3.5 Structural Analysis of Case 3 First and fourth rows of $\boldsymbol{H}_{3}$ becomes 0 , indicating that the saved motion will appear in neither systems. Since the situation is the same as bilateral control, whose connectivity matrix becomes the same as the hybrid matrix, which is used in bilateral control.
Table 3. Experimental parameters

\begin{tabular}{|c|l|c|}
\hline Parameter & Description & Value \\
\hline \hline$K_{\mathrm{p}}$ & P gain in position control & 1200.0 \\
$K_{\mathrm{d}}$ & D gain in position control & 420.0 \\
$C_{\mathrm{f}}$ & P gain in force control & 500.0 \\
$g_{\mathrm{d}}$ & CoF of DOB for a motor & $90.0 \mathrm{rad} / \mathrm{s}$ \\
$g_{\mathrm{r}}$ & CoF of RFOB for a motor & $90.0 \mathrm{rad} / \mathrm{s}$ \\
\hline
\end{tabular}

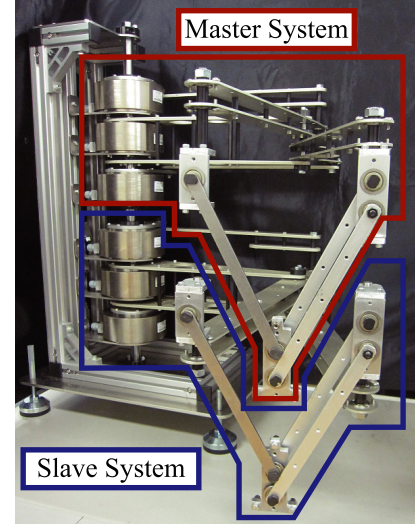

Fig. 5. Experimental setup

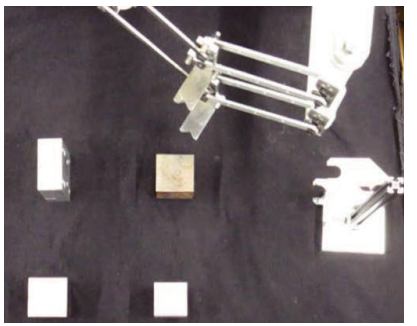

Fig. 6. Contact environment



Fig. 7. The trajectory of saved motion

\section{Experiments}

Experiments are conducted in this section to examine the validity of the proposed method.

4.1 Setup Controllers are placed in the joint space, and the gains for each controller are set to be all the same. The parameters commonly used in this section are shown in Table 3. The manipulator used in this experiment is shown in Fig. 5. Each system has three motors which allow operating the end effector in the cartesian coordinate. The motion stored in the database is the response of the master system given in Fig. 7. Motion started from the top left side and ended at the top right side in Fig. 7. At each vertex, the end effector was pushed to an aluminum block. The environmental setup is shown in Fig. 6 . The cases presented in the experiments are the same as the one shown in Section 3. $C_{\mathrm{pp}}$ is set as the same as $C_{\mathrm{p}}$ in Case 1.

4.2 Experimental Results The results of the proposed methods are shown in Figs. 8, 9, and 10. Because of the limitation of the paper length, every figure shows the result of joint 1 . Both responses are listed in the same figure to display the relationship between the responses between the 

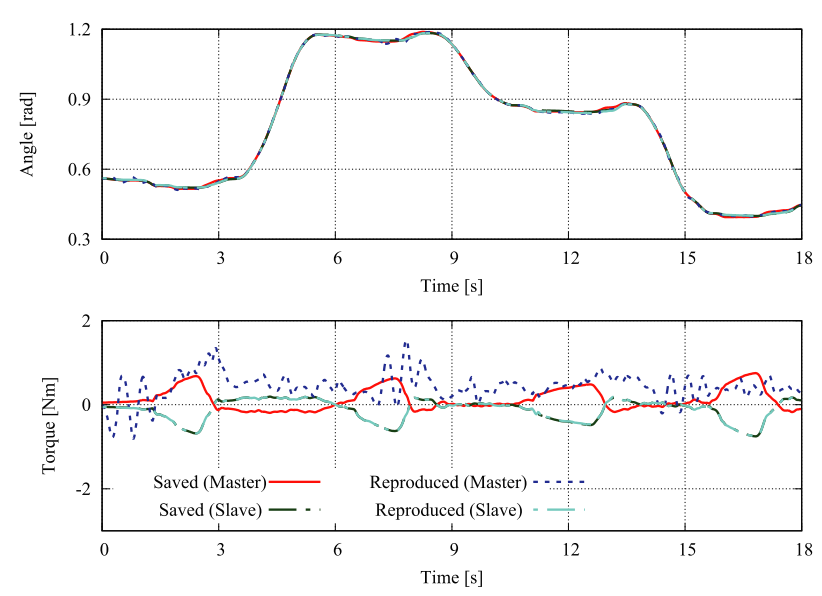

Fig. 8. Experimental results of Case 1 (joint 1)


Fig. 9. Experimental results of Case 2 (joint 1)
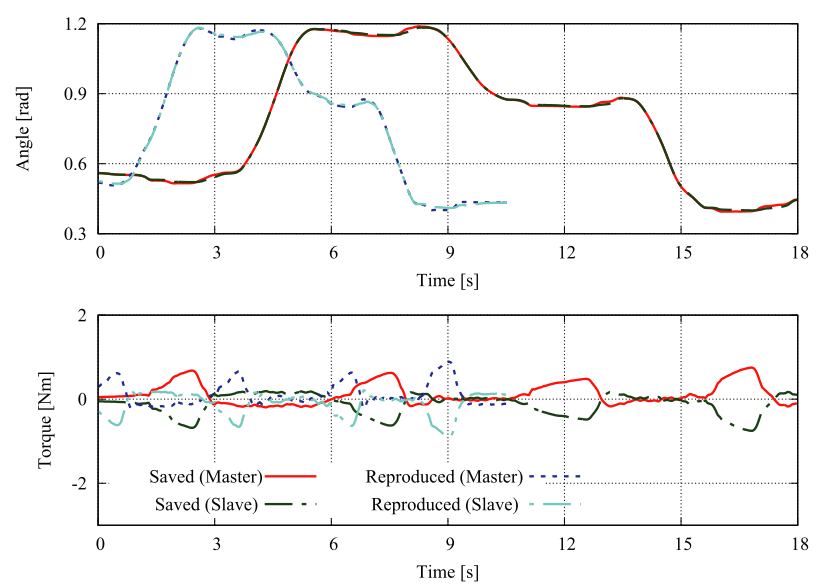

Fig. 10. Experimental results of Case 3 (joint 1)

saved phase and the reproduction phase.

The result of Case 1 is given in Fig. 8. Since $\beta$ is zero in this case, the motion of the master system in the reproduction phase will not affect the reproduced motion at all. The position responses of both master and slave systems become the same as those of the motion saving phase even though the different force is applied in the master system. All motion of every system follows the same trajectory. As for the force response, the response of the motion reproduction phase does not realize the law of action and reaction. This is because the force applied to the master system is not controlled. The
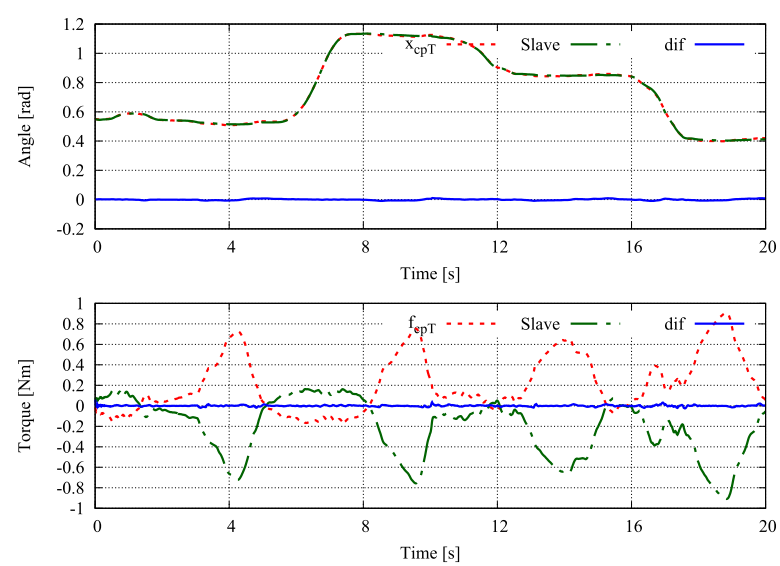

Fig. 11. Result of combined master motion and slave system motion in Case 2 (joint1)

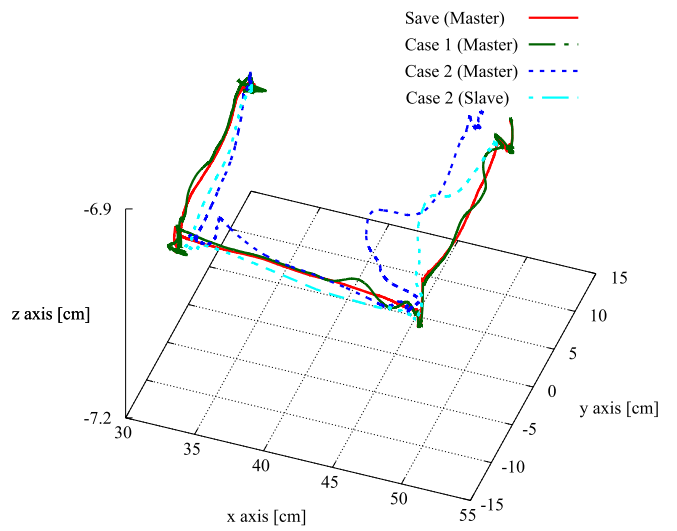

Fig. 12. The trajectory comparison between Case 1 and 2

force response in the reproduction phase well matches the result of the slave system in the saving phase.

The result of Case 2 is given in Fig. 9. This is the mixture between two motions, and it seems both control goals are not realized from the figure. However, since the relationship between the combined master system and the slave system is shown in (29), by plotting the response of the combined master system that is shown in Fig. 11, it can be seen that force and position errors are quite small. The mixed motion results in Case 2 can be obtained as

$$
f_{\mathrm{cpT}}^{2}=\frac{f_{\mathrm{m}}^{\mathrm{DB}}+f_{\mathrm{m}}}{2}, x_{\mathrm{cpT}}^{2}=\frac{x_{\mathrm{m}}^{\mathrm{DB}}+x_{\mathrm{m}}}{2} .
$$

Both trajectory tracking and realization of the law of action and reaction was realized.

The result of Case 3 is given in Fig. 10. In this case, the system becomes the same as bilateral control because the motion from the database is completely disconnected. In this experiment, the motion at the reproduction phase finished earlier than that of the saving phase. Even though the responses of the saved motion and that of the reproduced motion are different, it can be said that the desired control was realized.

To show the difference of the trajectory between that of Case 1 and Case 2, the trajectories in the workspace are listed in Fig. 12. In Case 1, since the force at the master system in the reproduction phase is not used as a control input, the trajectory tracks that of the saved motion. On the contrary, in Case 2, since the mixture of the saved motion and the motion 

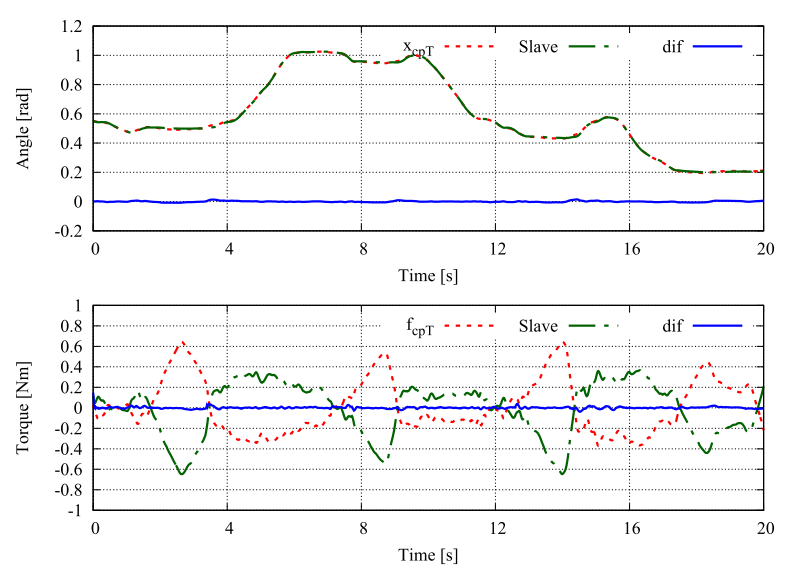

Fig. 13. Result of Case 2-1
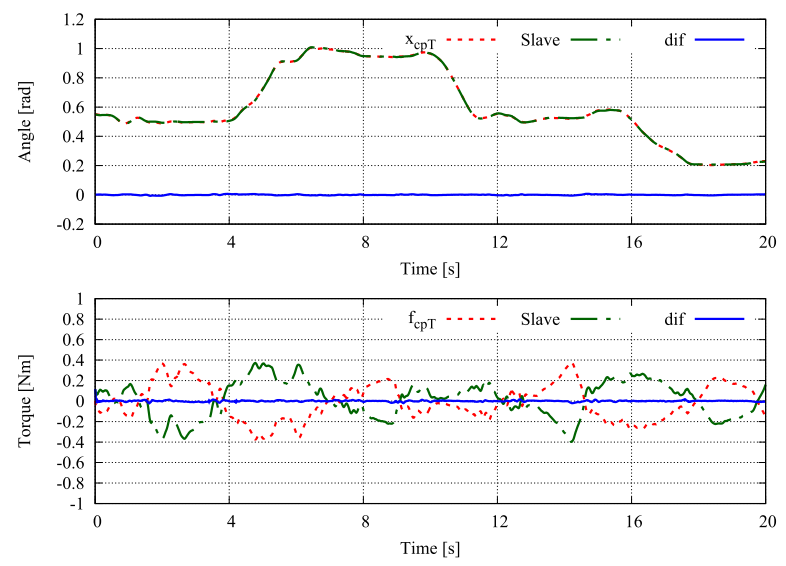

Fig. 14. Result of Case 2-2


Fig. 15. Result of Case 2-3

of the master system at the reproduction phase will be transformed by the matrix $\boldsymbol{T}$, the master motion does not track the response of the saving phase.

4.3 Comparison within Case 2 To further show how different the trajectories are when changing the reproduction ratio, the paper conducted additional experiments. $\alpha$ and $\beta$ are set as

$$
\begin{array}{ll}
\text { Case 2-1: } & (\alpha, \beta)=(1,1), \\
\text { Case 2-2: } & (\alpha, \beta)=(0.5,1), \\
\text { Case 2-3: } & (\alpha, \beta)=(1,0.5) .
\end{array}
$$

The joint space responses are shown in Figs. 13, 14, and 15. According to (29), the slave system tracks the responses
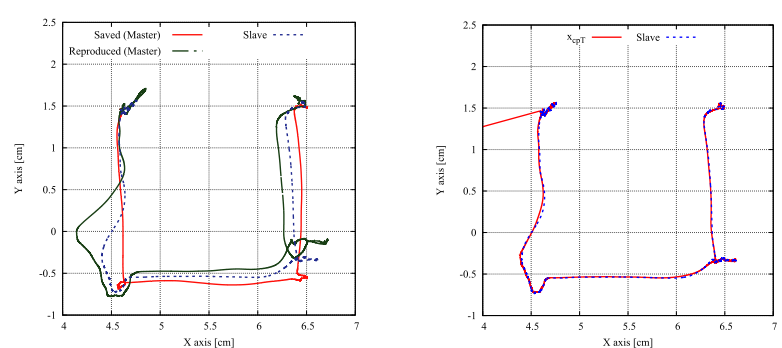

Fig. 16. Results of Case 2-1 Fig. 17. Modification of Fig. 16 in workspace
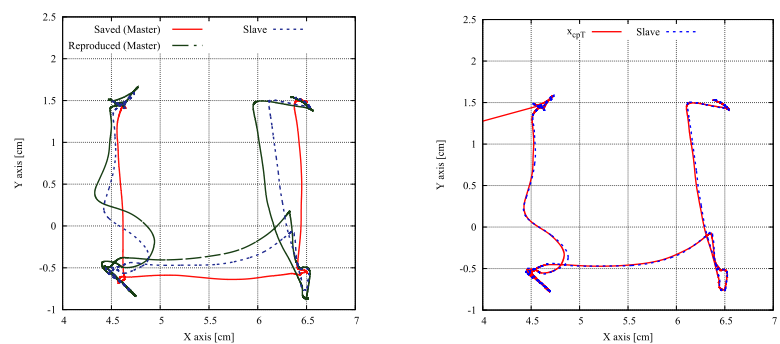

Fig. 18. Results of Case 2-2 Fig. 19. Modification of Fig. 18 in workspace
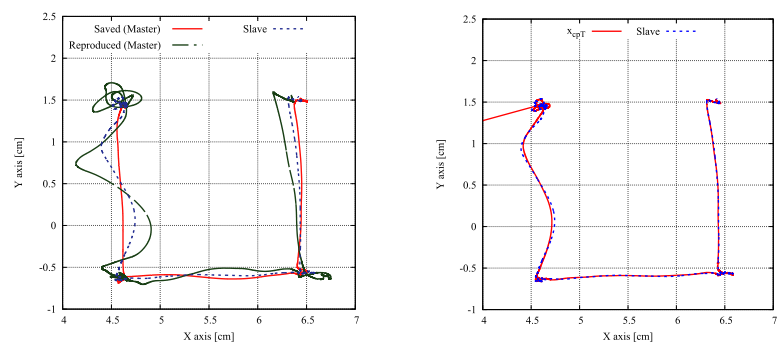

Fig. 20. Results of Case 2-3 Fig. 21. Modification of Fig. 20 in workspace

of $x_{\mathrm{cpT}}$ and $f_{\mathrm{cpT}}$, respectively. In these cases, the combined master system trajectory and force become

$$
\begin{aligned}
& x_{\mathrm{cpT}}^{2-1}=\frac{x_{\mathrm{m}}^{\mathrm{DB}}+x_{\mathrm{m}}}{2}, \quad x_{\mathrm{cpT}}^{2-2}=\frac{x_{\mathrm{m}}^{\mathrm{DB}}+2 x_{\mathrm{m}}}{3}, \quad x_{\mathrm{cpT}}^{2-3}=\frac{2 x_{\mathrm{m}}^{\mathrm{DB}}+x_{\mathrm{m}}}{3}, \\
& f_{\mathrm{cpT}}^{2-1}=\frac{f_{\mathrm{m}}^{\mathrm{DB}}+f_{\mathrm{m}}}{2}, \quad f_{\mathrm{cpT}}^{2-2}=\frac{f_{\mathrm{m}}^{\mathrm{DB}}+2 f_{\mathrm{m}}}{3}, \quad f_{\mathrm{cpT}}^{2-3}=\frac{2 f_{\mathrm{m}}^{\mathrm{DB}}+f_{\mathrm{m}}}{3} .
\end{aligned}
$$

The red lines in the figures are the responses of these variables. All of the results show that the error between the response of the slave system and that of the mixed master system is almost zero in all time. The results clearly show that the slave system tracks the motion of that of the mixed master system.

The trajectory results in the xy plane are shown from Figs. 16 to 21. The results of Figs. 17, 19, and 21 show the trajectory well matches between the two. Case 2-1 is the same as Case 2, and Case 2-2 is the situation where the reproduction ratio of the master motion in the reproduction phase is weak compared with the saved motion, and Case 2-3 is vice versa. During the experiments, a random force was intentionally applied to the master system to see how the applied force in the reproduction phase affects the reproduced motion.

As they can be seen from the results, the motion reproduced in the slave system depends on the values $\alpha$ and $\beta$. When $\alpha$ is set smaller than $\beta$, which is Case 2-2, the trajectory of the slave system becomes close to that of the saved 
motion. On the other hand, the slave system tries to track the motion of the master motion in the reproducing phase more when $\alpha$ is set larger than $\beta$.

\section{Conclusions}

In this paper, the hybrid motion reproduction method using a master/slave type motion-copying system was proposed. The setup used master-slave manipulators to obtain both applied force from the person in the motion reproduction phase and the force from the contact environment. It is essential to consider the mixture between the motion of data and human at the reproduction phase; therefore, the additional coordinate transformation matrix was proposed in this paper. Reproduced motion in the slave system was designed using the index reproduction ratio. By setting the ratio as 0 , motion from the person will not be reproduced in the slave system. The experimental results showed that the proposed method works under variable reproduction ratios.

One of the possible application of the proposed method is to use the system to motion training. Whether the quality of motion training improves or not will be examined in the future.

\section{Acknowledgment}

This work was partially supported by JSPS KAKENHI Grant Number $18 \mathrm{H} 03784$.

\section{References}

( 1 ) R.C. Goertz: “Mechanical master-slave manipulator", Nucleonics, Vol.12, No.11, pp.45-46 (1954)

( 2 ) P.F. Hokayem and M.W. Spong: "Bilateral teleoperation: An historical survey", Automatica, Vol.42, No.12, pp.2035-2057 (2006)

( 3 ) L. Eusebi and C. Melchiorri: "Force reflecting telemanipulators with timedelay: Stability analysis and control design", IEEE Transactions on Robotics and Automation, Vol.14, No.4, pp.635-640 (1998)

( 4 ) F. Mobasser, K. Hashtrudi-Zaad, and S. Salcudean: "Impedance reflecting rate mode teleoperation", in Proceedings of the IEEE International Conference on Robotics and Automation, ICRA, Vol.3, pp.3296-3302 (2003)

( 5 ) M. Tajiri, P. López, and Y. Fujimoto: "Design of two-channel bilateral control systems by a transfer-function-based approach", IEEE Transactions on Industrial Electronics, Vol.65, No.7, pp.5655-5664 (2018)

( 6 ) R. Kubo, N. Iiyama, K. Natori, K. Ohnishi, and H. Furukawa: "Performance analysis of a three-channel control architecture for bilateral teleoperation with time delay", IEEJ Journal of Industry Applications, Vol.127, No.12, pp.1224-1230 (2007)

( 7 ) D. Lawrence: "Stability and transparency in bilateral teleoperation", IEEE Transactions on Robotics and Automation, Vol.9, No.5, pp.624-637 (1993)

( 8 ) S. Katsura, W. Iida, and K. Ohnishi: "Medical mechatronics: An application to haptic forceps", Annual Reviews in Control, Vol.29, No.2, pp.237-245 (2005)

( 9 ) Y. Kuniyoshi, M. Inaba, and H. Inoue: "Learning by watching: extracting reusable task knowledge from visual observation of human performance", IEEE Journal of Robotics and Automation, Vol.10, No.6, pp.799-822 (1994)

(10) Y. Yokokura, S. Katsura, and K. Ohishi: "Stability analysis and experimental validation of a motion-copying system", IEEE Transactions on Industrial Electronics, Vol.56, No.10, pp.3906-3913 (2009)

(11) C. Fitzgerald: "Developing baxter", in Proceedings of the IEEE Conference on Technologies for Practical Robot Applications, TePRA, pp.1-6 (2013)
(12) W. Chung, C. Rhee, Y. Shim, H. Lee, and S. Park: "Door-opening control of a service robot using the multifingered robot hand", IEEE Transactions on Industrial Electronics, Vol.56, No.10, pp.3975-3984 (2009)

(13) Y. Jiang, Y. Zhu, K. Yang, C. Hu, and D. Yu: "A data-driven iterative decoupling feedforward control strategy with application to an ultraprecision motion stage", IEEE Transactions on Industrial Electronics, Vol.62, No.1, pp.620-627 (2015)

(14) H. Kuwahara, T. Shimono, H. Tanaka, D. Yashiro, and K. Ohnishi: "Abstraction of action components unconstrained by alignment of haptic sensing points", IEEE Transactions on Industrial Electronics, Vol.58, No.8, pp.31963204 (2011)

(15) T. Nozaki, T. Mizoguchi, and K. Ohnishi: "Motion-copying system with variable impedance based on scaled bilateral control in one-degree-of-freedom robot", Vol.3, No.1, pp.1-9 (2014)

(16) A. Matsui and S. Katsura: "Motion-copying system using modal information for motion reproduction", in Proceedings of the Annual Conference of IEEE Industrial Electronics Society, IECON, pp.6132-6137 (2013)

(17) K. Miura, A. Matsui, and S. Katsura: "Synthesis of motion-reproduction systems based on motion-copying system considering control stiffness", IEEE/ASME Transactions on Mechatronics, Vol.21, No.2, pp.1015-1023 (2016)

(18) S. Katsura: "Real-world haptics for supporting motion training", in Proceedings of the IEEJ Annual Meeting, pp.1-4 (2014)

(19) K. Ohishi, K. Ohnishi, and K. Miyachi: "Torque-speed regulation of DC motor based on load torque estimation method", in Proceedings of the IEEJ International Power Electronics Conference, IPEC, pp.1209-1218 (1983)

(20) T. Murakami, F. Yu, and K. Ohnishi: "Torque sensorless control in multidegree-of-freedom manipulator", IEEE Transactions on Industrial Electronics, Vol.40, No.2, pp.259-265 (1993)

Satoshi Nishimura (Member) received his B.E. degree in system


design engineering, M.E. and Ph.D. degrees in integrated design engineering from Keio University, Yokohama, Japan, in 2013, 2014, and 2017, respectively. His research interests include real-world haptics and motion control. He is a Member of IEEJ, as well as IEEE.

Seiichiro Katsura (Senior Member) received the B.E. degree in sys-

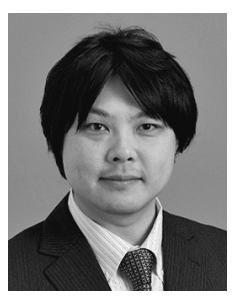
tem design engineering and the M.E. and Ph.D. degrees in integrated design engineering from Keio University, Yokohama, Japan, in 2001, 2002 and 2004, respectively. From 2003 to 2005, he was a Research Fellow of the Japan Society for the Promotion of Science (JSPS). From 2005 to 2008, he worked at Nagaoka University of Technology, Nagaoka, Niigata, Japan. Since 2008, he has been at Department of System Design Engineering, Keio University, Yokohama, Japan. Currently he is working as Professor. In 2017, he was a Visiting Researcher with the Laboratory for Machine Tools and Production Engineering (WZL) of RWTH Aachen University, Aachen, Germany. His research interests include applied abstraction, human support, data robotics, wave system, systems energy conversion, and electromechanical integration systems. Prof. Katsura serves as an Associate Editor of the IEEE Transactions on Industrial Electronics and Technical Editor of IEEE/ASME Transactions on Mechatronics. He was the recipient of The Institute of Electrical Engineers of Japan (IEEJ) Distinguished Paper Awards in 2003 and 2017, IEEE Industrial Electronics Society Best Conference Paper Award in 2012, and JSPS Prize in 2016. 